\title{
Redox Switches and Evolutionary Transitions
}

\author{
John F. Allen, Carol A. Allen, and Sujith Puthiyaveetil
}

\begin{abstract}
A redox switch is a signal transduction event or pathway that places gene expression under the regulatory control of the redox state of an electron carrier. Here we propose that the redox state of a quinone such as plastoquinone, ubiquinone or menaquinone is sensed and used as information to decide on the expression of genes for components of photosynthesis. Three quinone redox switches are proposed. The first, and most ancient, selects between transcription of type I and type II reaction centres. It is proposed that simple mutation in this redox switch produced constitutive expression of type I and type II reaction centres and so gave rise to the "Z-scheme" of oxygenic photosynthesis, where photosystem I and photosystem II are present in the same membrane at the same time, and are connected in series. The second redox switch controls the relative rates of synthesis of the reaction centres of photosystem I and photosystem II. This second switch is therefore responsible for control of photosystem stoichiometry. The need for continued operation of the second switch may have required the conservation of a chloroplast genome and chloroplast genetic system. The third redox
\end{abstract}

School of Biological and Chemical Sciences, Queen Mary, University of London, Mile End Road, London E1 4NS, UK switch governs post-translational modification of light-harvesting antenna proteins, and is responsible for complementary changes in the absorption cross-sections of photosystem I and photosystem II. There is extensive experimental support for the second and third redox switches, though the molecular mechanisms involved are uncertain. It is proposed that these three redox switches are based on bacterial two-component regulatory systems, are homologous, and that their application and modification produced the striking evolutionary transitions that flowed as consequences from the transition from anoxygenic to oxygenic photosynthesis, and from the transition from prokaryotic to eukaryotic cells.

Keywords Plastoquinone, ubiquinone, redox sensor, redox response regulator, oxygen evolution, chloroplast genome, state transitions, photosystem stoichiometry

\section{Introduction}

Reduction-oxidation or "redox" chemistry is the transfer of electrons or hydrogen atoms from a donor to an acceptor. In the course of the reaction, 
the donor, which reduces the acceptor, itself becomes oxidized. In the same reaction, the acceptor, which oxidises the donor, itself becomes reduced. Thus two redox couples are connected, and electrons flow from the more reducing couple to the more oxidizing couple.

Biological energy transduction involves chains of such redox couples, each couple acting as both donor and acceptor. In aerobic respiration, the initial electron donor in the respiratory electron transport chain is NADH or succinate, while the terminal electron acceptor is molecular oxygen, $\mathrm{O}_{2}$, which becomes reduced to water.

In oxygenic photosynthesis, two different, primary photochemical reactions co-operate in conversion of absorbed light energy. Together, the reaction centres oxidise the initial electron donor, water, and reduce the terminal electron acceptor, $\mathrm{NADP}^{+}$. Oxidation of water in photosynthesis produces the oxygen that is required for aerobic respiration, and that now occupies one-fifth of the Earth's atmosphere, by volume.

It is proposed that a redox switch that selected synthesis of one of two types of reaction centre became disabled by mutation, giving rise to the first cyanobacterium (Allen 2005; Allen and Martin 2007). The "redox switch hypothesis" also suggests an evolutionary origin for a sensor of the relative turnover of the two photochemical reactions of oxygenic photosynthesis. "State transitions" are a post translational modification of photosynthetic unit structure and function, serving to distribute absorbed light energy to the photochemical reactions in proportion to their capacity to use it (Allen 2003a). In state transitions, the ready-made redox switch, as a quinone redox sensor, was applied, adventitiously, to control activation of different mechanisms of distribution of light-harvesting function. In chloroplasts, the redox switch governs phosphorylation of membrane proteins and molecular recognition of the two photosystems by their shared, mobile light-harvesting complex.

Continued, additional application of the redox switch in gene expression may explain the persistence of genomes in both chloroplasts and mitochondria (Allen 1993a, b, 2003b). Chloroplasts exhibit the predicted redox control of gene expression (Allen and Allen 2007; Pfannschmidt et al. 1999; Puthiyaveetil and Allen 2007). Chloroplasts are now known to retain a bacterial redox sensor kinase, and this conserved and ancestral Chloroplast Sensor Kinase (CSK) is required for redox switching between transcription of chloroplast genes for the reaction centres of photosystem I and photosystem II (Puthiyaveetil and Allen 2007).

Redox switch 1: choosing between reaction centres

All known species of anaerobic phototrophs seem to have opted for specialisation to type I (typically photolithotrophic) or type II (typically photoorganotrophic) behaviour. However, photosynthetic bacteria are usually versatile in their ability to use energy, carbon, and electron sources. Adaptation - division of labour according to different environmental conditions - is common, if not universal. Therefore evolutionary divergence of type I and type II reaction centres may not have required loss of one type of reaction centre and its genes, and could have occurred within a single lineage of cells that retained different reaction genes for deployment in different environmental circumstances. Metabolic flexibility in anaerobic photosynthesis is particularly advantageous in environments with fluctuating supplies of $\mathrm{H}_{2} \mathrm{~S}$, such as hot springs, and in the vicinity of hydrothermal vents.

Figure 1 proposes that redox regulation of gene expression, under anaerobic conditions, determines whether type I or type II reaction centre genes are expressed in a single bacterial cell containing them. Quinone-level redox control of transcription of genes for type I reaction centres (RC I) and type II reaction centres (RC II) provides a suitable mechanism. Redox regulatory control of gene transcription is well established in both phototrophic and chemotrophic bacteria (Bauer et al. 1999, 2003; Eraso and Kaplan 2002; Oh and Kaplan 2000).

The emergence of two-photosystem, oxygenevolving photosynthesis from anaerobic, bacterial photosynthesis must have required either an anaerobic, two-photosystem precursor (Allen 2005), or else wholesale, lateral, and fully-functional gene 
transfer from one single-photosystem anaerobe into another.

Once a mechanism for water oxidation was in place, however inefficient, any mutation producing constitutive expression of both type I and type II genes would provide new functions for the two reaction centres. Their coupling to a single quinone pool allowed the reaction centres to function, for the first time, in series, and in cooperation: the acceptors for the type II centre, oxidising water, became the donors for the type I centre, reducing ferredoxin. This coupling provided the first oxygen-evolving bacteria with the advantages of both modes of photosynthesis (ATP synthesis and reduction of soluble electron carriers), and released them from dependency on transient supplies of $\mathrm{H}_{2} \mathrm{~S}$ for photoautolithotrophic growth. The novel electron donor, $\mathrm{H}_{2} \mathrm{O}$, was ubiquitous, and present at a concentration of $55.5 \mathrm{M}(1,000 / 18 \mathrm{~mole} / \mathrm{L})$. It is proposed that the origin of the "Z-scheme" of two light reactions, connected in series (Allen 2005; Allen and Martin 2007), occurred by means of a simple mutation that disabled the regulatory switch depicted in Fig. 1.

The selective advantage of using water as an electron donor might initially have been small, since the reaction would have been slow, and the product, molecular oxygen, was toxic. Subsequent selection, however, increased the redox midpoint potential of the primary (bacterio)chlorophyll electron donor, while more effectively coupling the manganese-containing water-oxidation complex to re-reduction of that donor, now observed in oxygenic photosynthetic organisms as the reaction centre chlorophyll $a, \mathrm{P}_{680}$.

Redox switch 2: adjusting the stoichiometry of photosystem I and photosystem II

It is suggested that direct redox control of expression of certain genes was present in the cyanobacterial progenitors of chloroplasts and was vital for selectively advantageous cell function before, during, and after the transition from bacterium to eukaryotic organelle. The mechanisms of this control have been conserved (Allen 1993a, 2003b).

Plastoquinone, an electron and hydrogen atom carrier located strategically between photosystems I and II of photosynthesis, exerts redox regulatory control over chloroplast transcription (Pfannschmidt et al. 1999), as outlined in Fig. 2. The direction in which the effect is exerted makes functional sense: reduction of plastoquinone increases photosystem I transcription and decreases photosystem II transcription; oxidation of plastoquinone decreases photosystem I transcription and increases photosystem
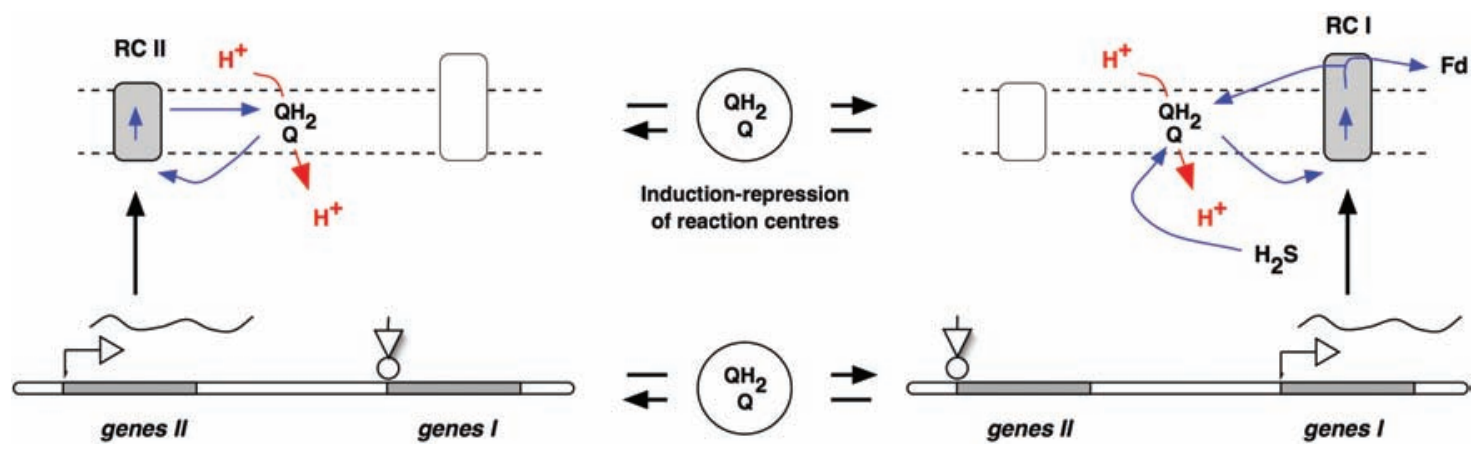

Fig. 1 Redox switch 1. Quinone-level redox control of gene expression in switching transcription between a single reaction centre of either type I or type II. This is the switch predicted to operate in protocyanobacteria. Protocyanobacteria are anoxygenic phototrophs able to adapt to different redox environments according to the presence of, for example, hydrogen sulphide as an electron donor. Failure of redox switch 1 produces constitutive expression of type I and type II reaction centres. In conjunction with a catalyst of water oxidation (Allen and Martin 2007), a mutation causing this redox switch failure gave rise to two photosystems, I and II, and to oxygenic photosynthesis (Allen 2005; Allen and Martin 2007; Allen and Puthiyaveetil 2005) 


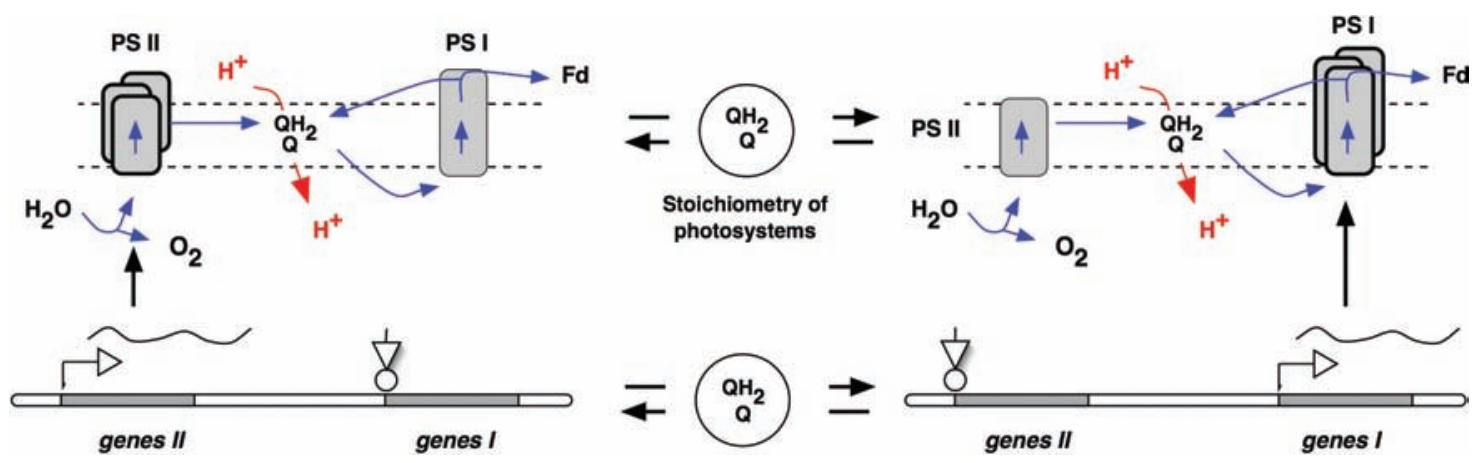

Fig. 2 Redox switch 2. Quinone-level redox control of gene expression in transcriptional adjustment of the stoichiometry of type I and II centres as photosystems I and II of cyanobacteria and chloroplasts. This is the switch that acts as a gain control determining the relative rates of synthesis, and therefore abundance, of photosystem I and photosystem II (Allen 1992; Pfannschmidt et al. 1999). Control of the stoichiometry of photosystem I and photosystem II is seen in all oxygenic phototrophs. Genes are retained in chloroplasts in order that this redox switch can operate upon them (Allen 1993a, 2003b; Allen et al. 2005). Gene and gene product are CO-located for Redox Regulation (CORR) (Allen 2003b)

II transcription. Since plastoquinone becomes reduced by the action of photosystem II and becomes oxidised by the action of photosystem I, redox control of chloroplast gene expression provides for a compensatory, self-adjusting response (Pfannschmidt et al. 1999), and provides part of an explanation for the ability of plants and algae to balance the ratio of the two photosystems despite changes in light quality.

Chloroplast plastoquinone redox control of transcription. This complementary, self-adjusting transcriptional response (El Bissati and Kirilovsky 2001; Li and Sherman 2000), suggests strongly that redox control of photosynthetic reaction centre gene transcription was present in the protochloroplast. Thus the mechanism of regulation is likely to have been conserved throughout the evolutionary transition from bacterial symbiont to cytoplasmic organelle. A conserved mechanism of redox regulation of transcription in photosynthetic prokaryotes (Bauer et al. 2003) and chloroplasts (Allen 1993c; Pfannschmidt et al. 1999), is predicted if evolutionary continuity of the process has been maintained.

Other possible precedents for redox signalling components of cytoplasmic organelles include the prr (Eraso and Kaplan 2002; Oh and Kaplan 2000) and reg (Bauer et al. 1999) redox regulatory systems of purple non-sulphur bacteria. The TspO "oxygen sensor" of Rhodobacter sphaeroides is present in mammalian mitochondria as a protein known there as a benzodiazepine receptor, and each protein will complement loss of the other (Yeliseev and Kaplan 2000).

Redox switch 3: adjusting the absorption crosssection of photosystem I and photosystem II

If chloroplasts inherited redox switch 2 from cyanobacteria, then, by the same argument, a functionally equivalent post-translational mechanism of redox control of light-harvesting function by photosystems I and II may have been conserved during the transition from prokaryote to eukaryotic subcellular organelle.

Protein phosphorylation is known to be involved in the plastoquinone redox switch that produces light-state transitions or chloroplasts and algae that contain the light-harvesting antenna protein LHC II (Allen 1992; Allen et al. 1981).

The broad outline of the mechanism that achieves redistribution of absorbed excitation energy is summarised in Fig. 3. When electrons pile up in plastoquinone, an electron carrier connecting photosystem I with photosystem II, then a protein kinase (Bellafiore et al. 2005) acts to phosphorylate the apoproteins of the light-harvesting chlorophyllprotein complex, LHC II. Upon phosphorylation, 


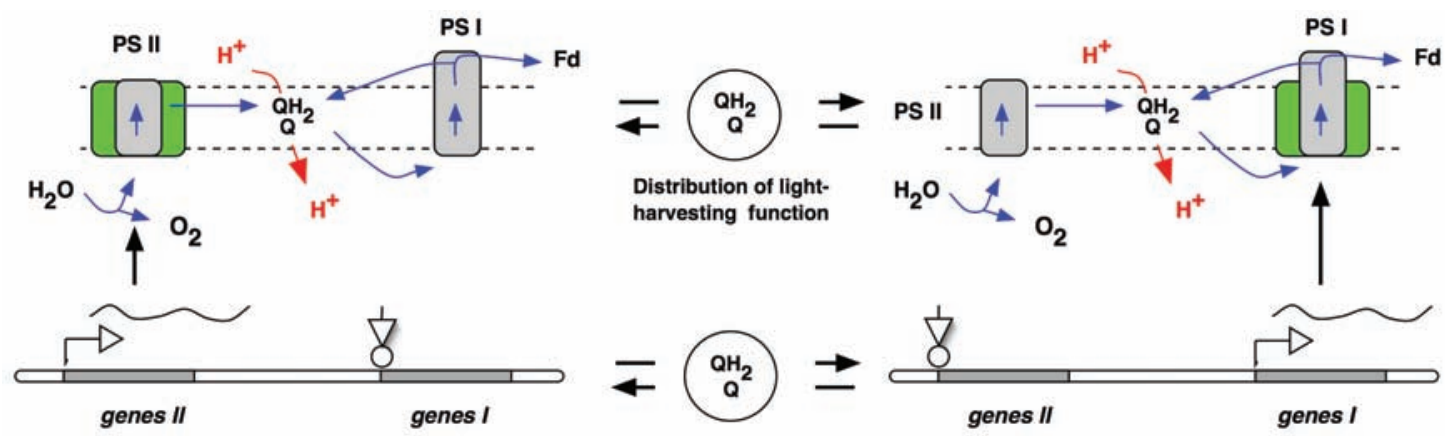

Fig. 3 Redox switch 3. Quinone-level redox control of gene expression in post-translational modification and distribution of light-harvesting function between photosystems I and II. This is the switch that underlies state 1-state 2 transitions - the selfadjustment of distribution of absorbed excitation energy between photosystem I and photosystem II (Allen 1992, 2003a). The target of the response is the peripheral and mobile light-harvesting complex. In cyanobacteria and red algae this is the phycobilisome. In chlorophytes, it is light-harvesting complex II (LHC II), which becomes phosphorylated when the redox switch reports to the LHC II kinase that plastoquinone is in its reduced form, plastoquinol

LHC II leaves photosystem II, and acts, instead, as the light-harvesting antenna for photosystem I. Activation of an LHC II kinase therefore decreases absorption of light by photosystem II and increases absorption of light by photosystem I.

Phosphorylation of LHC II is the basis of the transition to "state 2", a state of adaptation to photosystem II-specific light. The transition to state 2 clears the pile-up of electrons in plastoquinone in two ways: it decreases electron flow into plastoquinone from photosystem II, and increases electron flow out of plastoquinone to photosystem I. Conversely, a shortage of electrons in the plastoquinone pool between the photosystems is a signal that electrons are leaving faster than they enter. The LHC II kinase is then switched off, LHC II becomes dephosphorylated by a phospho-LHC II phosphatase, and the return of LHC II to photosystem II makes good the imbalance of energy distribution by driving a transition to "state 1 ".

\section{Discussion}

All three redox switches proposed here operate in response to a change in the redox state of a quinone pool. It is possible that a single quinone redox sensing mechanism has been conserved, and applied: (i) to reaction centre gene transcription as a purely "on-off" device (Fig. 1); (ii) to reaction centre gene transcription to adjust the relative rates of synthesis of the apoproteins of photosystem I and II reaction centres (Fig. 2), and (iii) post-translationally to control light-harvesting complex structure and relative affinity for photosystems I and II (Fig. 3).

Quinone redox state may be an over-riding important factor in cellular metabolism generally, since quinone pools serve as points of intersection of electron transport chains that are constructed of controllable modules whose stoichiometry relative to each other must be controlled in the interests both of thermodynamic efficiency and of safety.

Grasping new and unpredictable chemical opportunities to use free energy for metabolism and growth, cells have tinkered with redox switches and transformed both themselves and the geology of our planet (Dietrich et al. 2006; Russell et al. 2007; Russell and Hall 2006).

Acknowledgments. This work is supported by a Royal Society-Wolfson Research Merit Award to JFA.

\section{References}

Allen CA, Allen JF (2007) Redox effects on chloroplast protein synthesis and phosphorylation. In: Allen JF, Gantt E, Golbeck JH, Osmond B (eds) Photosynthesis. Energy from the Sun: 14th International Congress 
on Photosynthesis. Springer, Berlin/Heidelberg/New York, pp 903-907.

Allen JF (1992) Protein phosphorylation in regulation of photosynthesis. Biochim Biophys Acta 1098:275-335.

Allen JF (1993a) Control of gene-expression by redox potential and the requirement for chloroplast and mitochondrial genomes. J Theor Biol 165:609-631.

Allen JF (1993b) Redox control of gene-expression and the function of chloroplast genomes - an hypothesis. Photosynth Res 36:95-102.

Allen JF (1993c) Redox control of transcription - sensors, response regulators, activators and repressors. FEBS Lett 332:203-207.

Allen JF (2003a) Botany. State transitions - a question of balance. Science 299:1530-1532.

Allen JF (2003b) The function of genomes in bioenergetic organelles. Philos Trans R Soc Lond B Biol Sci 358:19-37.

Allen JF (2005) A redox switch hypothesis for the origin of two light reactions in photosynthesis. FEBS Lett 579:963-968.

Allen JF, Martin W (2007) Evolutionary biology: Out of thin air. Nature 445:610-612.

Allen JF, Puthiyaveetil S (2005) Chloroflexus aurantiacus and the origin of oxygenic, two-light reaction photosynthesis in failure to switch between type I and type II reaction centres. In: Est Avd, Bruce D (eds) Photosynthesis: Fundamental Aspects to Global Perspectives. Alliance Communications Group, Lawrence, KS, pp 753-756.

Allen JF, Bennett J, Steinback KE, Arntzen CJ (1981) Chloroplast protein-phosphorylation couples plastoquinone redox state to distribution of excitation-energy between photosystems. Nature 291:25-29.

Allen JF, Puthiyaveetil S, Strom J, Allen CA (2005) Energy transduction anchors genes in organelles. Bioessays 27:426-435.

Bauer CE, Elsen S, Bird TH (1999) Mechanisms for redox control of gene expression. Ann Rev Microbiol 53:495-523.

Bauer C, Elsen S, Swem LR, Swem DL, Masuda S (2003) Redox and light regulation of gene expression in photosynthetic prokaryotes. Philos Trans R Soc Lond B Biol Sci 358:147-154.
Bellafiore S, Barneche F, Peltier G, Rochaix JD (2005) State transitions and light adaptation require chloroplast thylakoid protein kinase STN7. Nature 433: 892-895.

Dietrich LEP, Tice MM, Newman DK (2006) The co-evolution of life and Earth. Curr Biol 16:R395-R400.

El Bissati K, Kirilovsky D (2001) Regulation of psbA and psaE expression by light quality in Synechocystis species PCC 6803. A redox control mechanism. Plant Physiol 125:1988-2000.

Eraso JM, Kaplan S (2002) Redox flow as an instrument of gene regulation. Methods Enzymol 348: 216-229.

Li H, Sherman LA (2000) A redox-responsive regulator of photosynthesis gene expression in the cyanobacterium Synechocystis sp. Strain PCC 6803. J Bacteriol 182:4268-4277.

Oh JI, Kaplan S (2000) Redox signaling: Globalization of gene expression. EMBO J 19:4237-4247.

Pfannschmidt T, Nilsson A, Allen JF (1999) Photosynthetic control of chloroplast gene expression. Nature 397:625-628.

Puthiyaveetil S, Allen JF (2008) A bacterial-type sensor kinase couples electron transport to gene expression in chloroplasts. In: Allen JF, Gantt E, Golbeck $\mathrm{JH}$, Osmond B (eds) Photosynthesis. Energy from the Sun: 14th International Congress on Photosynthesis. Springer, Berlin/Heidelberg/New York, pp 1181-1186.

Russell MJ, Hall AJ (2006) The onset and early evolution of life. In: Kesler SE, Ohmoto H (eds) Evolution of Early Earth's Atmosphere, Hydrosphere, and Biosphere Constraints from Ore Deposits. Geological Society of America, Memoir 198, pp 1-32.

Russell MJ, Allen JF, Milner-White EJ (2007) Inorganic complexes enabled the onset of life and oxygenic photosynthesis. In: Allen JF, Gantt E, Golbeck JH, Osmond B (eds) Photosynthesis. Energy from the Sun: 14th International Congress on Photosynthesis. Springer, Berlin/Heidelberg/New York, pp 1187-1192.

Yeliseev AA, Kaplan S (2000) TspO of Rhodobacter sphaeroides - a structural and functional model for the mammalian peripheral benzodiazepine receptor. J Biol Chem 275:5657-5667. 